\title{
PERIODIC FLUX VARIABILITY OF STARS DUE TO THE REFLEX DOPPLER EFFECT INDUCED BY PLANETARY COMPANIONS
}

\author{
Abraham Loeb ${ }^{1}$ and B. Scott Gaudi ${ }^{2}$ \\ Institute for Advanced Study, Einstein Drive, Princeton, NJ 08540; loeb@ias.edu, gaudi@ias.edu \\ Received 2003 March 10; accepted 2003 March 27; published 2003 April 8
}

\begin{abstract}
Upcoming space-based photometric satellites offer the possibility of detecting continuum flux variability at the micromagnitude ( $\mu \mathrm{mag}$ ) level. We show that the Doppler flux variability induced by the reflex motion of stars due to planetary companions has an amplitude of $(3-\alpha) K / c$, where $K$ is the reflex radial velocity amplitude and $\alpha \approx\left(d \ln F_{\nu} / d \ln \nu\right)$ is the logarithmic slope of the source spectral flux in the observed frequency band. For many of the known close-in planetary systems with periods $P \lesssim 0.2 \mathrm{yr}$, the periodic Doppler variability, $O(\mu \mathrm{mag})$, is significant relative to the variability caused by reflected light from the planetary companion. For companions with $P \gtrsim 0.2 \mathrm{yr}$, the Doppler signal is larger than the reflected light signal. We show that the future photometric satellites should reach the sensitivity to detect this Doppler variability. In particular, the Kepler satellite should have the photon noise sensitivity to detect at a signal-to-noise ratio $\gtrsim 5$ all planets with minimum mass $M_{p} \sin i \gtrsim 5 M_{\mathrm{J}}$ and $P \lesssim 0.1 \mathrm{yr}$ around the $\sim 10^{4}$ main-sequence stars with spectral types $\mathrm{A}-\mathrm{K}$ and apparent magnitude $V<12$ in its field of view.
\end{abstract}

Subject headings: planetary systems — techniques: photometric

\section{INTRODUCTION}

Planets outside the solar system orbiting main-sequence stars were first discovered in the mid-1990s (Mayor \& Queloz 1995; Marcy \& Butler 1996) through the small periodic reflex motion that they imprint on the radial velocity of their primary stars. The planetary systems discovered by the radial velocity surveys to date are qualitatively different from the solar system in important ways that were unexpected by earlier theoretical models of planet formation.

Although radial velocity studies have been highly effective at identifying more than a hundred extrasolar planetary systems, ${ }^{3}$ the method is inherently limited in several ways. First, generally it can be used only to find planets orbiting old latetype stars. Second, it is not sensitive to planets with mass $M_{p} \lesssim M_{\oplus}$. Finally, one can infer only the semimajor axis $a$, eccentricity, and a lower limit to the planet mass. It is therefore important to seek new complementary techniques that will allow better characterization of the physical properties of the known planets and extend the current sample to different types of stars and lower mass planets.

The power of combining the radial velocity method with other techniques was best illustrated in the case of the planetary companion to HD 209458. Originally discovered using radial velocity surveys, this planet was subsequently found to transit its parent star. Combination of the radial velocity data with the original ground-based transit data allowed the measurement of the mass (without an inclination angle ambiguity), radius, and density of this planet (Charbonneau et al. 2000; Henry et al. 2000). Further ultra-high-precision Hubble Space Telescope (HST) observations of several transits allowed the exquisite measurement of these planetary properties, as well as the detection of sodium lines in its atmosphere (Brown et al. 2001; Charbonneau et al. 2002). Perhaps the most exciting aspect of

\footnotetext{
${ }^{1}$ Guggenheim Fellow; on sabbatical leave from the Department of Astronomy, Harvard University, Cambridge, MA 02138.

${ }^{2}$ Hubble Fellow.

${ }^{3}$ See http://cfa-www.harvard.edu/planets/catalog.html for a list of planets and discovery references.
}

the HST measurements was the demonstration of the ability to achieve photometric precision at the level of $\sim 10^{-4}$ with spacebased observations.

In the future, several space-based photometric satellite missions are expected to reach a precision better than that demonstrated for HD 209458, down to the micromagnitude ( $\mu \mathrm{mag}$ ) level. These include Microvariability and Oscillations of STars (MOST), ${ }^{4} \mathrm{COn}$ vection, ROtation and planetary Transits (COROT) ${ }^{5}$ Kepler,${ }^{6}$ and Eddington. ${ }^{7}$ Scheduled for launch in 2003 June, MOST is a targeted mission, and among its primary targets are five stars with known extrasolar planetary companions. COROT, Kepler, and Eddington will monitor thousands of stars in one or several fields and search for very low-amplitude variability, as produced by stellar oscillations and planetary transits.

At the $\mu$ mag levels of variability probed by these satellites, many otherwise undetectable effects associated with planetary companions become significant. For transiting planets, the effects of the quadrupole moment of the parent star and more distant companions (Miralda-Escudé 2002), the oblateness of the planet (Hui \& Seager 2000; Seager \& Hui 2002), and the presence of moons and rings associated with the planet (Sartoretti \& Schneider 1999; Brown et al. 2001) are all potentially detectable. For both transiting and nontransiting companions, flux variations arising from tidal distortion of the parent star due to the planet (von Zeipel 1924; Drake 2003), back heating of the star from the planet (Green et al. 2003), and reflected and scattered light from the planet's atmosphere (Seager, Whitney, \& Sasselov 2000; Sudarsky, Burrows, \& Hubeny 2003; Green et al. 2003) may all be significant.

Here we examine another potential source of periodic flux variability, namely, the photometric flux variation of the primary star due to the Doppler effect. The reflex motion of the star induced by its planetary companion causes slight Doppler variations in its photometric flux. In $\S 2$, we derive the amplitude of this effect. In $\S 3$, we compare the Doppler effect with other

\footnotetext{
${ }^{4}$ See http://www.astro.ubc.ca/MOST.

${ }^{5}$ See http://smsc.cnes.fr/COROT.

${ }^{6}$ See http://www.kepler.arc.nasa.gov.

${ }^{7}$ See http://sci.esa.int/home/eddington/index.cfm.
} 


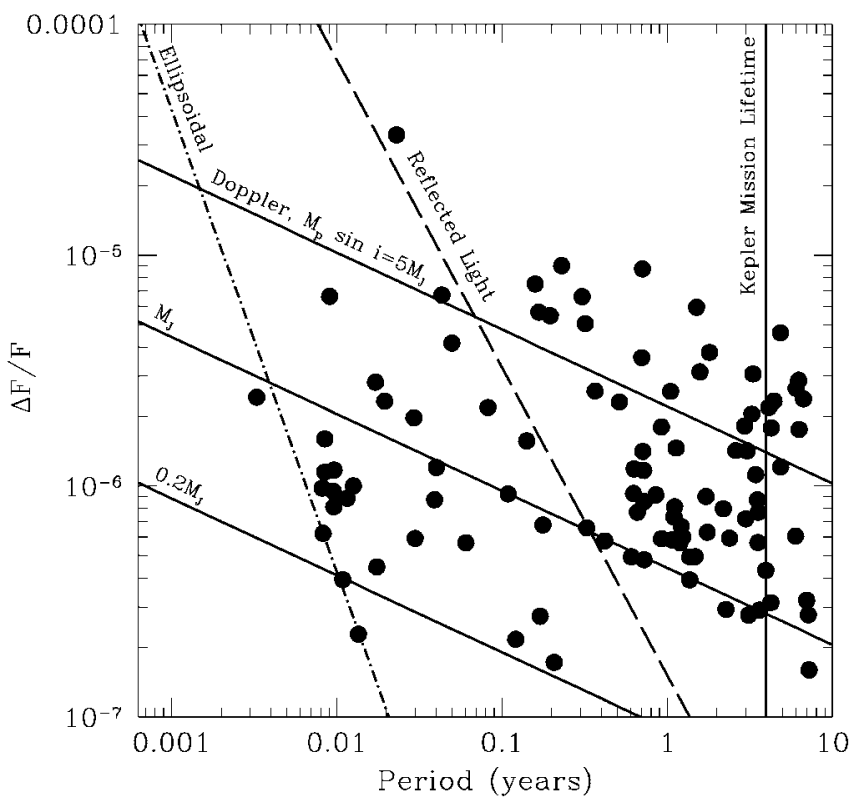

FIG. 1.-Expected flux variation amplitude as a function of the orbital period for the known extrasolar giant planets (filled circles). Also shown are the expected signals for fixed planet masses, assuming circular orbits, inclinations of $90^{\circ}$, and a solar-type primary. The dashed line shows the level of expected reflected light variations for a Jupiter-radius planet, with Lambert sphere scattering (albedo $=\frac{2}{3}$ ) and at full phase. The dash-dotted line shows the level of expected ellipsoidal light variations for a Jupiter-mass planet and solar-type primary. The solid vertical line is the Kepler mission lifetime (4 yr).

sources of stellar flux variability in the presence of planetary companions. In $\S 4$, we demonstrate that the Doppler variability should be detectable with the upcoming MOST and Kepler missions. Finally, we discuss the systematic errors and intrinsic variability in $\S 5$ and summarize our conclusions in $\S 6$.

\section{DOPPLER FLUX VARIATIONS}

A star moving at a radial nonrelativistic velocity $v_{r}$ relative to the observer obtains a Doppler shift to its bolometric flux by an amount (see eq. [4.97b] in Rybicki \& Lightman 1979, p. 141)

$$
F=F_{0}\left(1+4 \frac{v_{r}}{c}\right)
$$

where $c$ is the speed of light and the subscript 0 denotes observed quantities in the absence of the source motion. If the emitted flux per unit frequency scales as a power law in frequency, $F_{\nu 0} \propto \nu^{\alpha}$, then the Doppler shift in the observed frequency $\nu=\nu_{0}\left(1+v_{r} / c\right)$ implies that the observer detects

$$
F_{\nu}=F_{\nu 0}\left[1+(3-\alpha) \frac{v_{r}}{c}\right]
$$

The Doppler effect vanishes for $\alpha=3$, because $F_{\nu} / \nu^{3}$ is a relativistic invariant (corresponding to the phase-space density of photons). Therefore, $F_{\nu}=F_{\nu 0}$ for $\alpha=3$ irrespective of the source motion. Any other value of $\alpha$ leads to a net Doppler effect because of the change in the photon number flux.

For a general source spectrum, one can substitute $\alpha \approx$ $\left(d \ln F_{\nu 0} / d \ln \nu_{0}\right)$, where the derivative is averaged over the ob- served band of frequencies. Assuming a blackbody source spectrum with a temperature $T_{\text {eff }}$,

$$
\alpha(\nu) \simeq \frac{e^{x}(3-x)-3}{e^{x}-1},
$$

where $x \equiv h \nu / k T_{\text {eff }}$. In the Rayleigh-Jeans part of the spectrum $(x \ll 1), \alpha=2$, whereas in the Wien tail $(x \gg 1), \alpha=3-$ $x$. For solar-type stars $\left(T_{\text {eff }} \simeq 5700 \mathrm{~K}\right)$ and observations in the optical band $\left(\lambda \approx 600 \mathrm{~nm}\right.$ or $\left.\nu \approx 5 \times 10^{14} \mathrm{~Hz}\right)$, we get $\alpha \approx$ -1.3 . Given that the amplitude of the Doppler signal and the amount of flux received are both functions of frequency, the signal-to-noise ratio $(\mathrm{S} / \mathrm{N})$ will also depend on the observed frequency. For Poisson errors, the noise variance will be proportional to the square root of the flux. We find that the $\mathrm{S} / \mathrm{N}$ for $T_{\text {eff }} \sim 5700 \mathrm{~K}$ and a fixed logarithmic frequency interval peaks in the $V$ band, at $\lambda \sim 500 \mathrm{~nm}$ or $\nu \sim 6 \times 10^{14} \mathrm{~Hz}$.

\section{APPLICATION TO PLANETARY COMPANIONS}

The reflex motion of the star due to a planetary companion should produce periodic variations in its observed spectral flux. For circular orbits, the radial velocity of the star relative to the observer is simply $v_{r}=K \sin 2 \pi \phi$, where $\phi$ is the phase of the orbit and $K$ is the velocity semiamplitude,

$$
K=28.4 \mathrm{~m} \mathrm{~s}^{-1}\left(\frac{P}{1 \mathrm{yr}}\right)^{-1 / 3} \frac{M_{p} \sin i}{M_{\mathrm{J}}}\left(\frac{M_{*}}{M_{\odot}}\right)^{-2 / 3} .
$$

Here $M_{p}$ is the mass of the planet, $M_{\mathrm{J}}$ is the mass of Jupiter, $i$ is the inclination of the orbit, $M_{*}$ is the mass of the star, and we have assumed that $M_{p} \ll M_{*}$. Thus, the flux variability signal has a period $P$ and fractional amplitude $\Delta F / F_{0}=(3-$ $\alpha) K / c$. For orbits with an eccentricity $e$, the right-hand side of equation (4) should be multiplied by a factor of $\left(1-e^{2}\right)^{-1 / 2}$, which is typically very close to unity.

Since $K$ is a direct observable for companions detected spectroscopically via radial velocity variations, we can predict the amplitude of the flux variations for known planetary companions with essentially no ambiguity. Figure 1 shows the expected flux variation amplitude as a function of the orbital period for all the known radial velocity planets that have been detected so far, including the new transiting planet OGLE-TR-56 (Udalski et al. 2002; Konacki et al. 2003). We have estimated $T_{\text {eff }}$ values from the known spectral types of the host stars and assumed observations in the $V$ band. Also shown are the expected signals as a function of period for fixed planet masses, assuming circular orbits, inclination of $90^{\circ}$, solar-type primaries, and observations in the $V$ band.

The reflected light amplitude for a planet of radius $R_{p}$ is

$$
\frac{\Delta F}{F_{0}}=p\left(\frac{R_{p}}{a}\right)^{2} \text {. }
$$

Here $p$ and $R_{p}$ are the geometric albedo and the radius of the planet and $a$ is the semimajor axis of its orbit. The dashed line shows $\Delta F / F_{0}$ for Lambert sphere scattering $\left(p=\frac{2}{3}\right)$ and planets at full phase. Averaged over all phase angles, Lambert sphere scattering represents the upper limit to the amount of reflected light for a wide range of reasonable models, although larger scattered light signals are possible for phase angles near zero (when the planet is at superior conjunction) because of, e.g., strong backscattering (Seager et al. 2000). 
For short-period $(P \lesssim 0.2 \mathrm{yr})$ massive $\left(M_{p} \sin i \gtrsim M_{\mathrm{J}}\right)$ planets, the Doppler variability is expected to be a significant contaminant to the reflected light signal. For known radial velocity companions, the Doppler effect is completely predictable and its subtraction is straightforward. When searching for planets via reflected light, the magnitude of the Doppler effect will not be known a priori. However, since the reflected light signal will generally be largest when the planet is at superior conjunction, it will be $90^{\circ}$ out of phase with the Doppler signal, and it should be possible to cleanly disentangle the two signals. The Doppler signal is expected to dominate for long periods of $P \gtrsim 0.2 \mathrm{yr}$, since the reflected light signal falls off more rapidly with increasing orbital radius $\left(\propto a^{-2}\right)$ than the Doppler signal $\left(\propto a^{-1 / 2}\right)$.

There will also exist ellipsoidal light variations due to tidal effects on the star from the planet. We estimate the amplitude of these variations as

$$
\frac{\Delta F}{F_{0}} \sim \beta \frac{M_{p}}{M_{*}}\left(\frac{R_{*}}{a}\right)^{3}
$$

where $R_{*}$ is the radius of the primary and $\beta \simeq 0.45$ is the "gravity-darkening" exponent for solar-type stars. The amplitude of the expected ellipsoidal variations as a function of period is shown in Figure 1 as the dot-dashed line for $M_{p}=$ $M_{\mathrm{J}}, M_{*}=M_{\odot}$, and $R_{*}=R_{\odot}$. This effect should be subdominant for all but the shortest periods. Green et al. (2003) predict that back heating of the star by the planet should be an even smaller effect, $O\left(10^{-7}\right)$.

\section{DETECTABILITY}

For observations over a net duration $T$, the expected $\mathrm{S} / \mathrm{N}$ can be estimated as

$$
\frac{\mathrm{S}}{\mathrm{N}}=\frac{(3-\alpha)}{\sqrt{2}} \frac{K}{c} \sqrt{\Gamma T \times 10^{-0.4 \nu}}
$$

where $\Gamma$ is the number of photons per unit time collected by the instrument for a star of magnitude $V=0$, and we have assumed $P \ll T$. Note that the $\sqrt{2}$ factor is just the rms of the sinusoidal signal (for a planet in a circular orbit) in units of the amplitude. One can improve the $\mathrm{S} / \mathrm{N}$ by $\sqrt{2}$ if the period and phase of the signal are known in advance by making observations when the signal is at maximum.

The MOST satellite will collect $\Gamma \sim 1.6 \times 10^{8}$ photons s$^{-1}$ for a $V=0$ star (Green et al. 2003). In Figure 2, we show $M_{p} \sin i$ versus the period of the known extrasolar planets. Taking into account the $V$ magnitudes of the parent stars, we have calculated the expected S/N for a total of $100 \mathrm{hr}$ of integration with MOST. Since MOST is a targeted mission, we have assumed that the observations can be spaced over many periods and made at phases when the signal is largest. Thus we assume a $\mathrm{S} / \mathrm{N}$ that is a factor of $\sqrt{2}$ larger than given in equation (7). We have highlighted those stars for which $\mathrm{S} / \mathrm{N} \geq 3$. Of these, one is currently included in the MOST target list: $\tau$ Boo, for which $\mathrm{S} / \mathrm{N} \sim 6$ is expected.

The Kepler satellite will look at a single field continuously for $T=4 \mathrm{yr}$ and will collect $\Gamma \simeq 1.4 \times 10^{10}$ photons s$^{-1}$ for a $V=0$ star. The detection limits for primaries of spectral type $\mathrm{B}-\mathrm{K}$ are shown in Figure 2, assuming a total integration time of $T=4 \mathrm{yr}, V=12$, circular edge-on orbits, and $\mathrm{S} / \mathrm{N} \geq 5$ for detection. There are $\sim 10^{4}$ such main-sequence stars in Kepler's

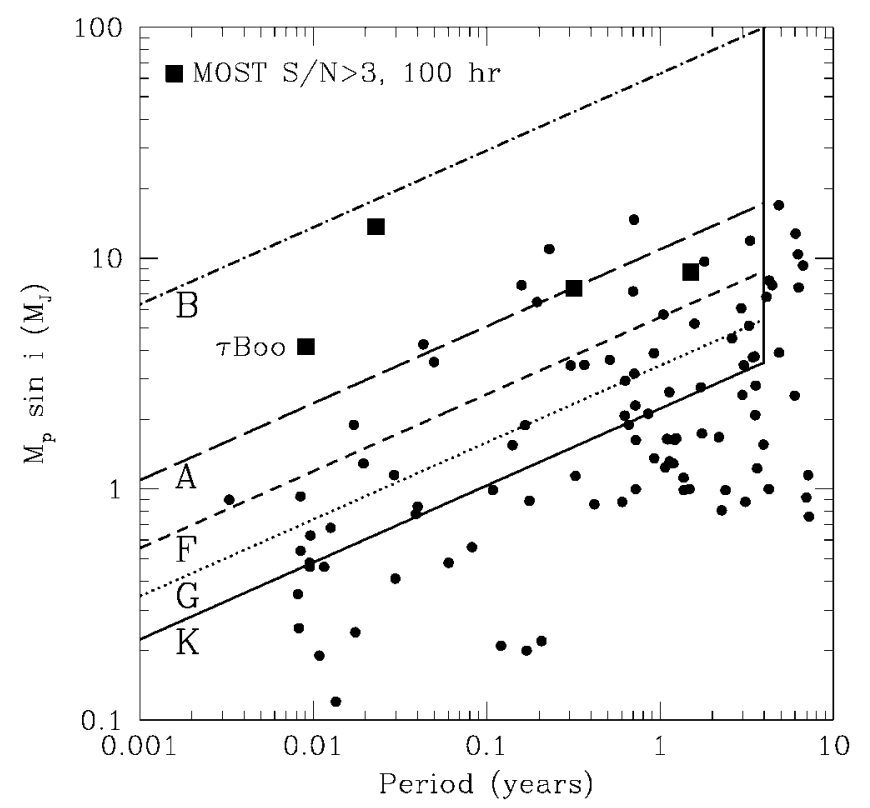

FIG. 2.-Mass as a function of orbital period for the known extrasolar giant planets (filled circles). The large filled squares show those known planets for which the $\mathrm{S} / \mathrm{N}$ of the flux variation is greater than 3 for $100 \mathrm{hr}$ of integration time with the MOST satellite. The lines show the $\mathrm{S} / \mathrm{N}>5$ detection limits for the Kepler mission for stars with $V=12$ and spectral types B, A, F, G, and $\mathrm{K}$ (top to bottom diagonal lines).

field of view. If the frequency of massive $\left(M \gtrsim 5 M_{\mathrm{J}}\right)$ shortperiod $(P \lesssim 0.1 \mathrm{yr}$ ) planets is $0.1 \%$, Kepler should detect $\sim 10$ such companions.

Both MOST and Kepler will have broad wavelength filters in order to gather as many photons as possible. MOST's bandpass will cover 350-700 nm, whereas Kepler's bandpass will cover $400-850 \mathrm{~nm}$. The spectra of the sources will vary considerably over these wavelength ranges, and our assumption of a fixed spectral slope appropriate to the center of the $V$ band will break down. We have estimated the magnitude of this effect by integrating the blackbody spectrum over a uniform bandpass from 400 to $800 \mathrm{~nm}$. We find that the expected $\mathrm{S} / \mathrm{N}$ is smaller by $\sim 5 \%$ than we have estimated before. The source spectra will deviate from the blackbody shape because of the presence of, for example, spectral lines. To determine the effects of such deviations, we have calculated the $\mathrm{S} / \mathrm{N}$ using a high-resolution solar spectrum of Kurucz et al. (1984), kindly provided to us by E. Turner. Again, we find that the difference from the naive calculation is small, $\sim 6 \%$.

\section{SYSTEMATIC ERRORS AND INTRINSIC VARIABILITY}

The amplitudes of the Doppler effects that we have considered are extremely small, even for the extremely precise upcoming space-based photometric missions. We therefore briefly consider both systematic errors and intrinsic variability.

The photometric stability requirements for the detection of the signals that we consider are considerably more stringent than the requirements for the detection of the signals that these satellites are designed to measure. To achieve the photon limit during the duration of the transit of an Earth-like planet, the photometry must be stable to roughly 1 part in $10^{5}$ over the $\sim 10 \mathrm{hr}$ duration of the transit. In contrast, we require that the photometry be stable to roughly 1 part in $10^{6}$ at least over the $\sim 3$ day periods of the smallest planetary orbits. Reflected light signals also require stability over similar timescales, but in the 
cases in which these signals are several times larger, the stability requirements are less severe.

Not much is known about intrinsic variability at the levels that we are considering for any stars except for the Sun. The intrinsic variability of the Sun is $\$ 10^{-5}$ on timescales of a few days or less (Batalha et al. 2001). This is of the order of the expected integrated Poisson error over these timescales and thus should not significantly affect our S/N estimates. However, the Sun does show significant (of the order of a few parts in $10^{4}$ ) variability on 30 day periods or longer (Batalha et al. 2001). This variability is a consequence of magnetic activity and sunspots. Depending on the coherence of its periodicity, this variability may complicate the detection of planets with longer periods via the Doppler variability. If measurements in two frequency bands are available, it may be possible to isolate the Doppler effect through its unique dependence on $\alpha$ (see eq. [2]).

\section{CONCLUSIONS}

Equation (2) provides the amplitude of the Doppler flux variability induced by the reflex motion of stars because of close-in planetary companions. For many of the known closein planetary systems, the Doppler variability will be similar or larger than the variability due to reflected light from the planetary companion (see Fig. 1).
We have found that future space-based photometry missions should reach the required photon-noise sensitivity for the detection of this Doppler effect. In particular, MOST should detect this effect due to the known companion of $\tau$ Boo at a S/N of $\sim 6$ in $100 \mathrm{hr}$ of integration. Kepler should detect all planetary companions with masses $\gtrsim 5 M_{\mathrm{J}}$ and periods $\lesssim 0.1$ yr to the $\sim 10^{4}$ main-sequence stars with $V<12$ in its field of view (see Fig. 2). This may uncover planets around massive primaries that are inaccessible to radial velocity surveys and too distant from their primary stars to be detected via reflected light.

We thank Andy Gould, Jon Jenkins, Sara Seager, Ed Turner, Josh Winn, and the referee, Ron Gilliland, for useful discussions and comments. A. L. acknowledges support from the Institute for Advanced Study at Princeton and the John Simon Guggenheim Memorial Fellowship. This work was also supported in part by NSF grants AST 00-71019 and AST 02-04514 and NASA grant ATP02-0004-0093 (for A. L.) and in part by NASA through a Hubble Fellowship grant from the Space Telescope Science Institute, which is operated by the Association of Universities for Research in Astronomy, Inc., under NASA contract NAS5-26555 (for S. G.). The NSO/Kitt Peak FTS data used here were produced by NSF/NOAO.

\section{REFERENCES}

Batalha, N. M., Jenkins, J., Basri, G. S., Borucki, W. J., \& Koch, D. G. 2001, in Stellar Structure and Habitable Planet Finding, ed. B. Battrick (Noordwijk: ESA), 35

Brown, T. M., Charbonneau, D., Gilliland, R. L., Noyes, R. W., \& Burrows, A. 2001, ApJ, 552, 699

Charbonneau, D., Brown, T. M., Latham, D. W., \& Mayor, M. 2000, ApJ, 529, L45

Charbonneau, D., Brown, T. M., Noyes, R. W., \& Gilliland, R. L. 2002, ApJ, 568,377

Drake, A. 2003, ApJ, in press (astro-ph/0301295)

Green, D., Matthews, J., Seager, S., \& Kuschnig, R. 2003, ApJ, submitted

Henry, G. W., Marcy, G. W., Butler, R. P., \& Vogt, S. S. 2000, ApJ, 529, L41

Hui, L., \& Seager, S. 2002, ApJ, 572, 540

Konacki, M., Torres, G., Jha, S., \& Sasselov, D. D. 2003, Nature, 421, 507
Kurucz, R. L., Furenlid, I., Brault, J., \& Testerman, L. 1984, Solar Flux Atlas from 296 to $1300 \mathrm{~nm}$ (Sunspot: NSO)

Marcy, G. W., \& Butler, R. P. 1996, ApJ, 464, L147

Mayor, M., \& Queloz, D. 1995, Nature, 378, 355

Miralda-Escudé, J. 2002, ApJ, 564, 1019

Rybicki, G. B., \& Lightman, A. P. 1979, Radiative Processes in Astrophysics (New York: Wiley)

Sartoretti, P., \& Schneider, J. 1999, A\&AS, 134, 553

Seager, S., \& Hui, L. 2002, ApJ, 574, 1004

Seager, S., Whitney, B. A., \& Sasselov, D. D. 2000, ApJ, 540, 504

Sudarsky, D., Burrows, A., \& Hubeny, I. 2003, ApJ, in press (astro-ph/ 0210216)

Udalski, A., et al. 2002, Acta Astron., 52, 1

von Zeipel, H. 1924, MNRAS, 84, 665 